3. Baum, M., \& Houghton, J. Informed consent and controlled trials. Lancet, 1988, 2, 1194.

4. Faulder, C., Peckham, M. J., Baum, M., Bullimore, J. A., Cuzick, J., Durrant, K. R., Maguire, G. P., Sloane, J., Teasdale, C., Tobias, J. S., \& White, H. Informed consent: Ethical, legal and medical implications for doctors and patients who participate in randomised clinical trials. British Medical Journal, 1983, 286, 1117-21.

5. McPeek, B., Mosteller, F., \& McKneally, M. Randomized clinical trials in surgery. International Journal of Technology Assessment in Health Care, 1989, 5, 317-332.

6. Marshall, T., \& Moodie, P. Scrutinising research ethics committees. British Medical Journal, 1988, 297, 753.

7. Thomas, E. Informed consent. Lancet, 1986, 2, 1280.

8. Wittes, R. E., \& Friedman, N. A. Accrual to clinical trials. Journal of the National Cancer Institute, 1988, 80, 884-85.

\title{
EXPENSIVE MEDICAL AND SURGICAL TECHNOLOGY
}

\author{
Douglas Black \\ Royal College of Physicians of London
}

The very title - "Expensive Medical and Surgical Technology"- expresses in coded form a myth that is widely prevalent among those who consider the political and economic aspects of health care. Strangely, it is a myth that finds favor mainly at the extreme ends of the range of attitudes toward health care. Monetarists see it as an incentive toward increasing private provision of health care, while extreme egalitarians see it as another example of unjust privilege. The content of the myth is that there is a definable group of costly procedures, which can somehow be isolated from the general practice of medicine and surgery, after which such procedures can either be made the subject of special private provision or, alternatively, discarded from a rationed system of health care.

This myth is dangerous (1) and contains significant fallacies:

1. It implies that there is a category of technology that is somehow separable from the rest of medical and surgical practice.

2. It takes full account of the cost of process, while leaving out of the account the value of outcome.

3. It exaggerates the contribution made by so-called high technology to the total cost of health care.

4. It suggests that specious forms of managerial control are possible and important, thereby undermining the professional approach to the definition of priorities and the delivery of health care.

Pervasiveness and Continuity of Technology. There are two reasons why we should not isolate a particular group of procedures labeled "expensive medical and surgical techniques." First, technology is only a part of the practice of medicine. It varies in 
its relative importance according to the nature of the medical problem. In some situations, the "tecr:nology" is simply that of history-taking and competent physical examination. In other situations, the solution to the problem may require whole-body scanning or a complex surgical procedure. But even in these instances, the application of high technology only follows, and does not precede, the clinical definition of the problem.

Is it possible, or sensible, to make a distinction between "expensive" and "inexpensive" technical procedures? It is clearly possible, at the level of accountancy, to name a figure, for instance $£ 1,000$, above which the unit cost of a procedure is "expensive." But an arbitrary limit of this type would only make sense if "cost" and "waste" were synonymous. They are not. It is actually more wasteful, albeit cheaper, to carry out an inappropriate inexpensive procedure than an appropriate, costly one.

Cost of Process and Value of Outcome. A deceptively easy approach to the economic assessment of a service is simply to discover what it costs. But that method is a matter of accountancy and not a worthwhile economic analysis. A further step is the analysis of process, in which the various procedures involved in the delivery of health care are examined to determine whether they are being economically carried out. This is a useful exercise, if critically performed, and it is sometimes equated with cost-benefit analysis. But it is inherently incomplete, since it stops short at the level of the cost and efficiency of the means to an end, and it does not consider whether that end has been achieved. The desired ends of medical care are the prevention of illness and disability in the healthy population and the preservation of life and the relief of suffering in those who are sick.

There is, however, an important difference in the economic analysis of preventive and of therapeutic measures. The "outcome" of preventive measures has to be assessed in populations, and it generally becomes apparent only after the lapse of considerable time. It may follow that to increase relative expenditure on preventive measures, as is so often advocated, has to a certain extent to be either a matter of faith, as with some dietary measures, or else justified by retrospective analysis of the type that revealed the link between smoking and lung cancer. These assumptions are often confirmed later by prospective analysis of defined populations.

In contrast, the outcomes of therapeutic actions generally concern individuals and usually become apparent soon after the intervention, at least in acute illnesses. Therefore, they are more open to prompt economic analysis, comparing cost to outcome. That is not to say that such an analysis is easy, especially when the criteria by which outcomes are judged go beyond simple mortality or survival and take account of quality of life. Yet the choice of a new treatment, or a comparison of alternative modes of treatment, is incomplete without such an analysis. It is, for example, naive to point to the high cost of computerized tomography of the brain without also considering the additional diagnostic precision that it may bring to therapeutic decisions, while at the same time relieving the patient of the discomfort and even danger of angiography or air encephalography. It is equally naive to point to the high cost of a femoral popliteal graft without considering an amputee's loss in earnings, costs of converting his or her home for a wheelchair, the cost of a prosthetic limb, and the impact of amputation on the quality of life.

Exaggerated Estimate of the Cost of "Expensive" Technology. Obsession with the costs of "high" or "expensive" technologies obscures the fact that they account 
for only a very small part of the total expenditure on health care. Much more is spent on "low technology," i.e., the profusion of simple diagnostic and therapeutic techniques. Moreover, the costs of a service are overwhelmingly those of wages and salaries, of which only a small proportion is attributable to the acolytes of "high technology."

The Managerial Fallacy. The escalation of health care has led in some countries to panic solutions. One remedy has been to institute a commercial style of management. This approach is characterized by: an emphasis on cost; a shift away from the dominance of the medical profession; and hierarchical, as opposed to consensus, management. Managers tend to concentrate on process, which may seem easier for them to understand, and generally shy away from outcomes, which would require professional assessment. The fruits of this approach are sometimes bizarre: Beds are emptied and wards closed to lessen expense, but at the real cost of diminishing the service that is the raison d'être of the entire operation; or, patients are, at one time, detained to maintain "bed-occupancy," and, at another, hustled out to increase "turnover." In these and other ways, care is diminished, and incidental opportunities for teaching medical and nursing students are lost.

"Expensive technology," so far from being a disadvantage or even a disproportionate economic burden, is, in fact, something that can influence outcome favorably, and it is indeed inseparable from the general process and progress of medicine and surgery. But advocacy of existing and new technology does not imply that it must be allowed to run riot, and we must turn to consider possible measures of control.

Given the assumption - a realistic one in even the wealthiest of countries - that the resources available for health care will inevitably fall short of the demands made upon them and that the cost of new technology will represent a significant part of health expenditure, although still a small part in comparison with wages and salaries, there must be a critical assessment of any new technology before its use is made general within the health care delivery system. Before considering the criteria on which such an assessment should be made, we should address two questions:

1. At what stage in the development of a new technology, whether surgical, medical, or diagnostic, should assessment be made?

2. What might be an appropriate mechanism for making it?

Stage of Assessment. The first stage in the development of a technology is represented by the perception that there are both a need and a possible means of satisfying it or, alternatively, that a technique developed in another field of science may have an application to medicine or surgery. Pilot studies of two kinds then have to be carried out (a) to discover whether the proposed apparatus actually works and fulfills the purpose to which it is addressed and (b) to discover its acceptability both to health professionals and, more important, to patients. When satisfactory pilot studies have demonstrated the promise of an invention, pressures build up to have it made universally available. The right time for assessment of a new technology comes between the demonstration that it is practicable and making it generally available. Too early a critical assessment might stifle inventiveness before the full potential of a discovery has been appreciated, whereas assessment at too late a stage might fail to prevent dissemination of a technology that was no better or perhaps even worse than what was already available.

Mechanism of Assessment. Formal procedures of assessment have been devel- 
oped in the United States with an Office of Technology Assessment housed in the Congress and a well-established series of consensus conferences on both new and existing practice, including the technological aspects. Although the King Edward's Hospital Fund in the United Kingdom has begun to experiment with consensus conferences, the pleas of Sir Andrew Kay (2) and others for a formal control mechanism for new technologies remain unanswered. At the time when I knew it, some 10 years ago, there was a Supplies Division within the Department of Health and Social Security that was responsible for controlling the spread of new technologies throughout the National Health Service. Their assessment, however, tended to concentrate on the efficiency of the apparatus and on its possible commercial exploitation, rather than on where it stood in relation to what was already available, or whether there was a clinical need for the proposed device. The fact that something works cannot stand alone in establishing its usefulness, although it is, of course, a step in the right direction. As in other contexts, the ultimate decision should reflect the views of users and clients at least as much as those of producers. Both as users and as proxy customers, doctors must be involved in making the decisions.

Criteria of Assessment. Barbara Stocking and S. L. Morrison (3) have outlined the considerations that should have governed the introduction of whole-body scanning by computerized tomography. Technology may be either diagnostic or therapeutic, and the latter is at least superficially the easier to assess, since it can be made the subject of a controlled trial, provided that appropriate outcome measurements can be devised. But when the outcome is not a "cure," but the "answer to a question," the analysis becomes more complicated. It must include the specificity and the sensitivity of the method and the likelihood of random error. It must involve comparison with alternative methods of obtaining the same information. To give a specific example, the analysis of CT scanning 10 years ago could only compare it with existing $x$-ray imaging, whereas it would now have to be compared with nuclear magnetic resonance (NMR). Since, at the pragmatic level, diagnosis is not an end in itself, but a necessary preliminary to an assessment of the therapeutic options, it is also legitimate and, indeed, necessary to consider to what extent the information obtained will actually affect the choice and outcome of the therapy. An innovation that contributes materially to the treatment of a common clinical problem should clearly command some priority in comparison with an innovation that is relevant only to the treatment of a rare disease. Where the need for a procedure is unusual, central rather than local provision may be appropriate. The apparent cost of a new technology may be lessened by economies of scale as it becomes generalized within one country or captures overseas markets.

There is no special legitimacy to be attached to a hierarchy of technologies, whether "high" or "low," "expensive" or "inexpensive." The touchstone of choice must be what is most appropriate for the individual patient in each specific clinical situation. There is also, of course, an economic dimension, whether for the patient directly or for a tax-funded or insurance-based system. These points can perhaps be best illustrated by the example of the options available for the management of end-stage renal failure. End-stage renal failure arises when kidney function has declined to $5 \%$ or less of the normal level, and all reversible causes of renal failure have been ruled out. The main options, in order of ascending cost, are continuous ambulant peritoneal dialysis (CAPD), renal transplantation, hemodialysis (HD) at home, and HD in the hospital. 
In terms of quality of life, a successful transplant is generally (i.e., by patients as well as by surgeons) considered to be the best option. But even apart from the shortage of suitable kidneys, not every patient is suitable to receive a renal graft, so the other options must remain.

To look at a narrower choice, that between HD at home and HD in the hospital: the former has an annual cost only about half that of the latter and it relieves the patient from having to visit the hospital several times each week. On the other hand, the lower cost of HD at home is in part achieved by a transfer of the "social cost" of care from the hospital to the family, and, of course, there are households in which home dialysis is not a realistic possibility. The higher cost of hospital dialysis is partly attributable to the selection for treatment in the hospital of patients with catabolic or other complications that require more intensive dialysis and possibly other expensive treatments.

I hope this example demonstrates that the nature of the available technology, although it may be an important element, is still only one component of a complex decision, in which major weight must also be given to the clinical situation and to the wishes of the informed patient.

Full-scale technological analysis may have to be limited to major innovations until experience has been gained and resources have been made available. It should also be realized that the clinical and economic impact of an innovation, and its effect on long-term outcomes, may be fully apparent only after the lapse of time. Another consideration, perhaps a marginal one, is the effect of philanthropic provision of an apparatus that turns out to be costly to staff or to maintain or to have little effect on outcomes.

In summary, new developments in technology, as applied to medical and surgical practice, are to be encouraged, which is just as well, since they are probably inevitable. But they also call for critical assessment and, perhaps, a measure of formal control. It is, however, important that the criteria for their acceptance (or rejection) should not be limited to economic considerations but should also include their clinical applicability and their likely effect on outcomes.

\section{REFERENCES}

1. Jennett, B. High technology medicine: Benefits and burdens. Oxford: Oxford University Press, 1986.

2. Kay, A. W. Research in medicine (Rock Carling Monograph). London: Nuffield Provincial Hospitals Trust, 1977.

3. Stocking, B., \& Morrison, S. L. The image and the reality: A case-study of the impacts of medical technology. Oxford: Oxford University Press, 1978. 